\title{
Proportion and factors associated with low fifth minute Apgar score among singleton newborn babies in Gondar University referral hospital; North West Ethiopia.
}

\author{
Temesgen Worku Gudayu
}

Department of Midwifery, College of Medicine and Health Sciences, University of Gondar, Ethiopia

\begin{abstract} in Gondar University referral hospital; North West Ethiopia. with low $5^{\text {th }}$ minute Apgar score. augmented labor and low birth weight.

Keywords: Apgar score, Gondar University referral hospital.

DOI: https://dx.doi.org/10.4314/ahs.v17i1.2 abs.v17i1.2

\section{Introduction}

Childbirth is the period of increased risk of mortality for a mother and her baby ${ }^{1}$. An estimated $42 \%$ of the world's 535, 900 annual maternal deaths are intrapartum-related; these deaths are closely linked to the deaths of 1.02 million babies during labor and 904,000 intrapartum related ("birth asphyxia") neonatal deaths ${ }^{1-3}$.
\end{abstract}

Back ground: New born babies with low Apgar scores are at an increased risk of perinatal morbidity and mortality.

Objective: To assess proportion and factors associated with low $5^{\text {th }}$ minute Apgar Apgar score among singleton newborn babies

Methods: A cross-sectional study was conducted on singleton 261 live births from March - May, 2013. Data was collected from mother/newborn index using a structured and pre-tested questionnaire. It was then cleaned, coded and entered using EPI INFO version 3.4.3, then analyzed with IBM SPSS statistics versions 20.0. Logistic regression was used to identify significant variables

Result: The proportion of low $5^{\text {th }}$ minute Apgar score in this study was $13.8 \%$. Factors that were significantly associated with low $5^{\text {th }}$ minute Apgar score were: non-vertex fetal presentation, prolonged labor, presence of meconium stained liquor, induced/

Conclusion: Mainly obstetric factors contribute to low Apgar score. Improving labor management through implementing regular use of partograph, 1:1 midwife-client ratio and advanced electronic fetal monitoring technology is recommended.

Cite as: Gudayu TW. Proportion and factors associated with low fifth minute Apgar score among singleton

newborn babies in Gondar University referral hospital; North West Ethiopia. Afri Health Sci. 2017;17(1): 1-6. https:/ / dx.doi.org/10.4314/

The Apgar score, first introduced by Virginia Apgar ${ }^{4}$ in 1952 , initially was a practical method of systematically assessing newborn infants immediately after birth to help identify those requiring resuscitation ${ }^{5}$. Later, the Apgar scores determined at five minutes after delivery became

\section{Corresponding author:}

Department of Midwifery,

College of Medicine and

Health Sciences,

University of Gondar, Ethiopia

Tel: +251911755087

E-mail: teme.worku@gmail.com
Temesgen Worku Gudayu,

widely used for the prediction of asphyxia as well as hypoxic-ischemic encephalopathy and cerebral palsy ${ }^{6-9}$.

It is established that there is association between low Apgar scores and increased perinatal morbidity and mortality $^{10}$. A recent study showed that Apgar scores of less than 7 at five minutes after birth were associated with low cognitive function, neurologic disability and even subtle cognitive impairment as measured by academic performance at 16 years of age $\mathrm{e}^{11,12}$. However, the value of the Apgar score $<7$ is still considered controversial by different studies and many neonatologists across the world ${ }^{13,14}$. Perinatal morbidity and mortality can be reduced if high risk infants can be identified and managed appropriately. The present study therefore aimed to assess the proportion and associated factors with low $5^{\text {th }}$ minute Apgar score among newborns in Gondar University referral hospital.

\section{Methods}

An institution based cross-sectional study was conducted at the University of Gondar referral hospital, maternity ward from March-May, 2013. 
The hospital is located in North Gondar zone at about 727 kilometers NorthWest of Addis Ababa, the capital city of Ethiopia. The hospital provides delivery services 24 hours a day, 7 days a week and is staffed with midwives, intern doctors, general practitioners and obstetricians.

All mother/neonate index singleton live births after 28 weeks of gestation during the study period were included in the study. Deliveries of unknown gestational age(unknown last normal menstrual Period and no ultrasound estimation) were not included.

A sample size of 261 was calculated using single population proportion formula assuming a $35.7 \%$ proportion of $5^{\text {th }}$ minute low Apgar score from previous study in Ethiopia ${ }^{15}$ at a $95 \%$ confidence limit, $5 \%$ margin of error and correction formula.

Systematic random sampling technique was used to reach at each participant. By taking a monthly average of 350 deliveries from preceding year report and considering a skip interval of 4 , the calculated sample size was achieved in the three months of data collection period.

Data was collected by five graduating first class degree midwifery students in the delivery and operation rooms. Data collectors were assigned at day and night rotations to address the design. Training on the standard procedures of Apgar score estimation with emphasis on a three scale easily identifiable selection criteria for each Apgar score variables and an interview procedure was provided to data collectors. Each newborn recruited was assessed for Apgar in the $1^{\text {st }}, 5^{\text {th }}$ and $10^{\text {th }}$ minutes after birth and weighed only once soon after delivery. Maternal and obstetric information was collected at admission, during first stage of labor and after delivery.

The interview questionnaire consisted of maternal socio-demographic and obstetric variables. In addition, variables related to the newborn were included. Completeness of the data and relative accuracy of Apgar score estimation was evaluated by a senior midwife who was the maternity ward head on daily basis.
Apgar score was estimated using five variables [Strength and regularity of heart rate; (100 beats/minute or more (2 points), Less than 100 (1 point), none (0 points)), Lung maturity / breathing effort; (Regular breathing (2 points), Irregular and $<30$ breath/minute ( 1 point), None $(0$ points), Muscle tone and movement; (Active (2 points), Moderate (1 point), Limp (0 points)), Skin color / oxygenation; (Pink ( 2 points), bluish extremities (1 point), totally blue (0 points) and reflex response to irritable stimuli; (Crying (2 points), whimpering (1 point), silence ( 0 points). Then the score of each finding was summed up by investigator and a score below value seven was considered as low ${ }^{4-6}$.

All collected questionnaires were checked for completeness and consistency of responses manually. Then data was coded, entered in to EPI INFO version 3.4.3 and analyzed using IBM SPSS statistics versions 20.0. Bivariate crude odds ratio and multivariate adjusted odds ratio was done by Logistic regression model. The presence and strength of association of variables was assessed using odds ratio with $95 \%$ confidence interval.

Ethical clearance was obtained from department of Midwifery which is a delegate of the institutional review board of Gondar University. Permission to conduct the study was also obtained from Gondar University referral hospital. Participants were informed about the purpose and objective of the study. They were also informed that, they had the right to discontinue or refuse to participate in the study. Verbal consent was obtained from each study participants. Confidentiality of information and privacy was observed.

\section{Result}

A total of 261 neonate/mother pairs were involved in this study. About $82 \%$ of the mothers were aged $20-$ 34 years, with a range between 18 - 42 years and a median age of 25 years. Nearly three quarters were urban dwellers. Majority (95.0\%) were married and 55.9\% house wives (Table 1). 
Table 1: Socio-demographic characteristics of mothers who gave birth in Gondar University Referral Hospital, Gondar Town, Ethiopia; March - May, 2013

\begin{tabular}{|c|c|c|}
\hline Variable (characteristics) & Frequency(No) & Percent (\%) \\
\hline \multicolumn{3}{|l|}{ Age (maternal) } \\
\hline$<20$ & 18 & 6.9 \\
\hline $20-34$ & 215 & 82.4 \\
\hline $35+$ & 28 & 10.7 \\
\hline \multicolumn{3}{|l|}{ Marital status } \\
\hline Married & 248 & 95.0 \\
\hline Other * & 13 & 5.0 \\
\hline \multicolumn{3}{|l|}{ Occupation } \\
\hline House wife & 146 & 55.9 \\
\hline Government employee & 46 & 17.6 \\
\hline Private employee & 17 & 6.5 \\
\hline Merchant & 24 & 9.2 \\
\hline Others** & 28 & 10.8 \\
\hline \multicolumn{3}{|l|}{ Educational status } \\
\hline No formal education & 95 & 36.4 \\
\hline Primary education (1-8) & 53 & 20.3 \\
\hline Secondary education $(9-12)$ & 76 & 29.1 \\
\hline College and above & 37 & 14.2 \\
\hline \multicolumn{3}{|l|}{ Residence } \\
\hline Urban & 192 & 73.6 \\
\hline Rural & 69 & 26.4 \\
\hline \multicolumn{3}{|l|}{$\begin{array}{l}\text { Household Income per month } \\
\text { (per quartile) }\end{array}$} \\
\hline$<1000$ birr $(<\mathrm{Q} 1)$ & 64 & 24.5 \\
\hline $1000-2800$ birr (Q1-Q3) & 131 & 50.2 \\
\hline$>2800$ birr $(>\mathrm{Q} 3)$ & 66 & 25.3 \\
\hline
\end{tabular}

One hundred and thirteen mothers (43.3\%) were primi- in about $90 \%$ these mothers labor duration was less than parous. About $88 \%$ participants had history of at least one ANC follow up during the current pregnancy. In about $90 \%$ of the mothers labor was spontaneous and 24 hours. Nearly three quarter $(74.7 \%)$ of mothers had spontaneous vertex deliveries (SVD). The proportion of low $5^{\text {th }}$ minute Apgar score in newborn babies was $13.8 \%$ (Table 2).

Table 2: Obstetric and Neonatal characteristics of study participants in Gondar University Referral Hospital, Gondar Town, Ethiopia; March - May, 2013

\begin{tabular}{|c|c|c|}
\hline Variable & Frequency & Percent (\%) \\
\hline \multicolumn{3}{|l|}{ ANC follow up } \\
\hline Yes & 230 & 88.1 \\
\hline No & 31 & 11.9 \\
\hline \multicolumn{3}{|l|}{ Parity * } \\
\hline 1 & 113 & 43.3 \\
\hline $2-4$ & 135 & 51.7 \\
\hline$\geq 5$ & 13 & 5.0 \\
\hline \multicolumn{3}{|l|}{ Condition of labor } \\
\hline Spontaneous & 234 & 89.7 \\
\hline Induced/Augmented & 27 & 10.3 \\
\hline \multicolumn{3}{|l|}{ Duration of labor } \\
\hline 3-24hrs ( normal) & 234 & 89.7 \\
\hline$>24 \mathrm{hrs}$ ( prolonged ) & 27 & 10.3 \\
\hline \multicolumn{3}{|l|}{ Mode of delivery } \\
\hline SVD & 195 & 74.7 \\
\hline Instrumental & 15 & 5.7 \\
\hline $\mathrm{C} / \mathrm{S}$ & 51 & 19.6 \\
\hline \multicolumn{3}{|l|}{ Gestational Age at Birth } \\
\hline Preterm (<37 wks) & 20 & 7.7 \\
\hline Term (37-42 wks) & 232 & 88.9 \\
\hline Post term ( $>42 \mathrm{wks})$ & 9 & 3.4 \\
\hline \multicolumn{3}{|l|}{ Birth weight } \\
\hline Low $(1500-2500 \mathrm{gm})$ & 31 & 11.9 \\
\hline Normal (2500-4000 gm) & 230 & 88.1 \\
\hline \multicolumn{3}{|l|}{$5^{\text {th }}$ Minute APGAR Score } \\
\hline Low $(<7)$ & 36 & 13.8 \\
\hline Normal $(\geq 7)$ & 225 & 86.2 \\
\hline \multicolumn{3}{|l|}{ Sex } \\
\hline Male & 145 & 55.6 \\
\hline Female & 116 & 44.4 \\
\hline
\end{tabular}


The results of multivariate analysis (Table 3)indicated a significant association between fetal presentation and low $5^{\text {th }}$ minute Apgar score. Fetuses who adapted non-vertex presentation were 4.46 times $(\mathrm{AOR}(\mathrm{CI})=4.46(1.41$, 14.08)) more likely to have a low Apgar score than those who adapted vertex presentation.
Other factors that remained significantly associated on multivariate analysis include; induced/augmented labor, labor duration $>24$ hours, the presence of meconium and low birth weight (Table 3).

Table 3: Bivariate and multivariate analysis of factors associated with low $5^{\text {th }}$ minute Apgar score; Gondar Town, Ethiopia; March - May, 2013

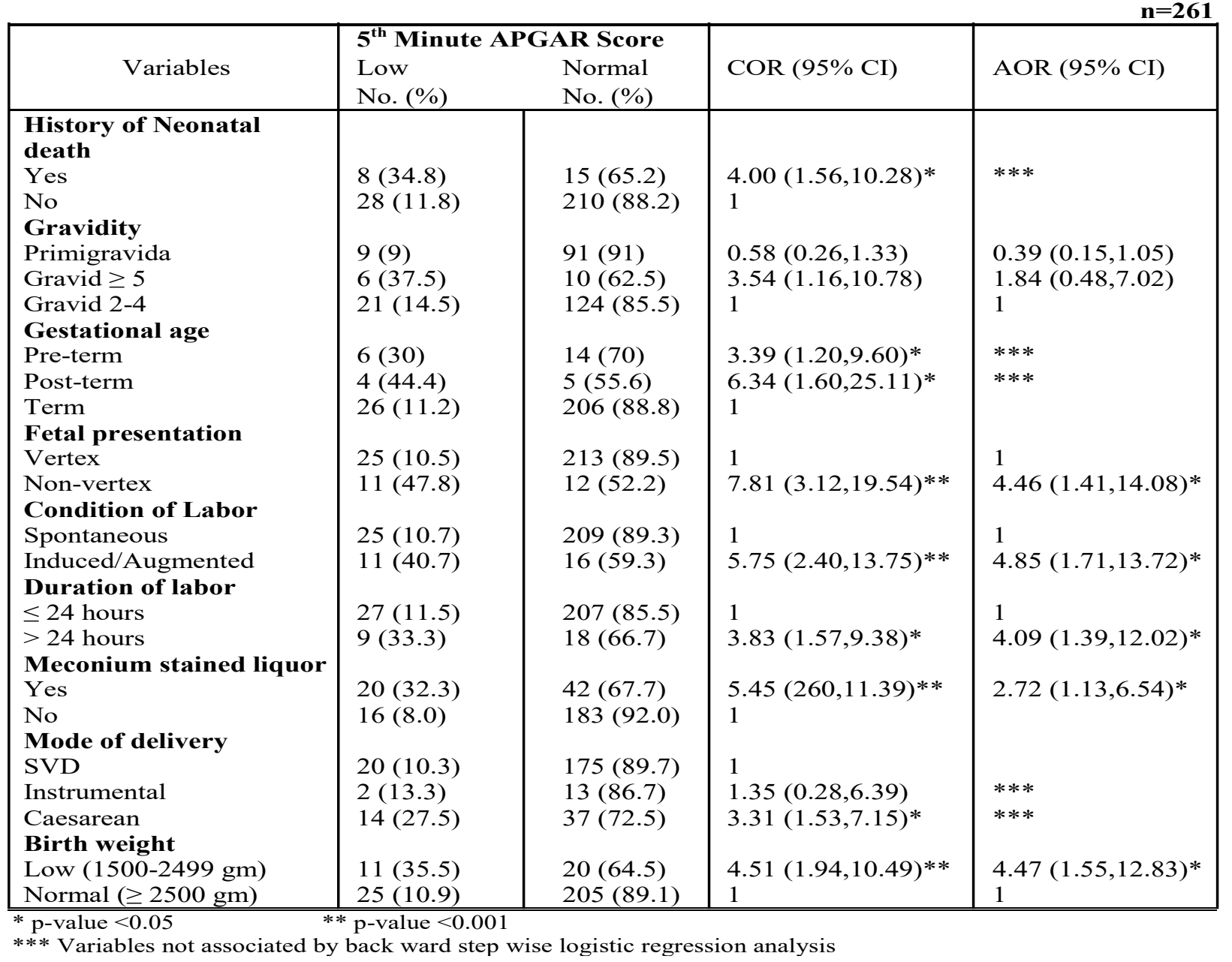

\section{Discussion}

The purpose of this study was to assess the proportion and associated factors of low $5^{\text {th }}$ minute Apgar score among newborns in Gondar University referral hospital. The proportion of low $5^{\text {th }}$ minute Apgar score in this study was $13.8 \%$. This result is lower than another institution-based study in South-West Ethiopia $(35.7 \%)^{15}$. This difference might be explained by the time gap between these two studies. In the earlier time, institutional delivery was only utilized by a small (5\% with $31-39.5 \%$ urban residents) proportion of the population in the country ${ }^{25,26}$; from this we can extrapolate that the then institutional deliveries might be self referred obstetric complications with high neonatal morbidity.

In other studies done among West, North and sub-Saharan African emigrants and in Uganda ${ }^{16,17} ; 3.9 \%, 1.8 \%$ and $8.4 \%$ proportion of low $5^{\text {th }}$ minute Apgar score was reported. The disparity in the proportion from current study might be due to better utilization of institutional delivery $^{27}$ (36.6\% with $79.2 \%$ urban residents) and probably better obstetric care even in the case of emigrants.

In the analysis of associated factors, non-vertex fetal presentation was significantly associated with low $5^{\text {th }}$ minute 
Apgar score. A study done in Uganda ${ }^{17}$ showed similar findings. This similarity might be due to the fact that in non-vertex fetal presentation, there will be chance of caesarean $^{18}$ or manipulative vaginal deliveries, which could further affect Apgar score.

One of the factors that were associated with $5^{\text {th }}$ minute low Apgar score in this study, prolonged labor, was similar to other studies ${ }^{19}$. In addition, induced/augmented labor and the presence of meconium stained liquor in this study were significantly associated with low Apgar score. In the case of prolonged labor, induction/augmentation and meconium stained liquor, there will be a chance of fetal distress, which could affect mode of delivery and subsequently distressed fetuses will have low Apgar scores. Studies also supported that tachy-systole and uterine rupture are among identified side effects of induction/augmentation of labor which can result in fetal distress, then passage of meconium and eventually end up in caesarean delivery or hysterectomy ${ }^{20,21}$.

The current study also revealed that newborn babies with low birth weight were about 4.5 times more likely to have low $5^{\text {th }}$ minute Apgar score compared to those with normal birth weight. The findings from other studies ${ }^{18,22}$ also showed consistent results with current study. Different from the finding in this study, one study ${ }^{23}$ showed low Apgar score associated with extremely low birth weight. This could be explained by the fact that small babies might suffer from difficult birthing and might develop difficulty in cardiopulmonary transition and perinatal asphyxia ${ }^{24}$.

\section{Limitations}

This study did not consider some potential risk factors for low Apgar score such as placental factors, multiple pregnancy, congenital syndromes and intra-uterine infections since the conditions were unavailable at the time of study.

\section{Conclusion and recommendation}

In this study $13.8 \%$ neonates had low Apgar score at $5^{\text {th }}$ minute of life. Mainly obstetrics factors (non-vertex fetal presentation, prolonged labor, presence of meconium stained liquor, induced/augmented labor and low birth weight) were significantly associated with low $5^{\text {th }}$ minute Apgar score. Improving labor management through implementing regular use of partograph, 1:1 midwife-client ratio and advanced electronic fetal monitoring technology is recommended. In addition, in order to address other potential variables and to identify immediate and long term outcomes of low apgar score, a large scale study is recommended.

\section{Acknowledgment}

I am highly indebted to College of Medicine \& Health Sciences, University of Gondar, for providing ethical clearance. I would like to extend my thanks to Gondar University referral hospital maternity ward staff for permitting me to conduct the study and providing the necessary preliminary information while conducting this study. I would also like to extend my appreciation to the study participants, supervisors and data collectors.

\section{Competing interests}

The author declares no competing interests.

\section{Author's contributions}

TW designed the study, participated in the data collection, performed analysis and interpretation of data, drafted the paper developed and revised the manuscript.

\section{References}

1. Lawn JE, Lee AC, Kinney M, et al. Two million intrapartum stillbirths and neonatal deaths: where, why, and what can we do? Int J Gynecol Obstet 2009;107:S5-S19.

2. Lawn J, Shibuya K, Stein C. No cry at birth: global estimates of intrapartum stillbirths and intrapartum-related neonatal deaths. Bull WHO 2005;83(6): 409-17. PubMed 3. Hill K, Thomas K, AbouZahr C, et al. Estimates of maternal mortality worldwide between 1990 and 2005: an assessment of available data. Lancet 2007;370(9595):1311_ 9. PubMed

4. Apgar, V. A proposal for a new method of evaluation of the newborn infant. Current Research in Anaesthesia 1953; 32:260-267.

5. Apgar, V. and James, L.S. Further observations on the newborn scoring system. Amer. J. Dis. Child. 1962; 104:419-428.

6. Casey BM, McIntire DD, Leveno KJ. The continuing value of the Apgar score for the assessment of newborn infants. N Engl J Med. 2001;344:467-71 PubMed .

7. Harrington DJ, Redman CW, Moulden M, et al. The long-term outcome in surviving infants with Apgar zero at 10 minutes: a systematic review of the literature and hospital-based cohort. Am J Obstet Gynecol. 2007;196:463 e1-5.

African Health Sciences Vol 17 Issue 1, March, 2017 
8. Thorngren-Jerneck K, Herbst A. Low 5-minute Apgar score: a population based register study of 1 million term births. Obstet Gynecol. 2001;98:65-70 PubMed .

9. Ehrenstein V, Pedersen L, Grijota M, et al. Association of Apgar score at five minutes with long-term neurologic disability and cognitive function in a prevalence study of Danish conscripts. BMC Pregnancy Childbirth. 2009;9:14.

10. Paul V.K., Singh M., Sundaram K.R., et al. Correlates of mortality among hospital - born neonates with birth asphyxia. Natl. Med. J. India. 1997; 10:54-57.

11. Ehrenstein V, Pedersen L, Grijota M, et al. Association of Apgar score at five minutes with long-term neurologic disability and cognitive function in a prevalence study of Danish conscripts. BMC Pregnancy and Childbirth. 2009;9 (14).

12. Andrea Stuart, Petra Otterblad Olausson, Källen K. Apgar Scores at 5 Minutes After Birth in Relation to School Performance at 16 Years of Age. The American College of Obstetricians and Gynecologists Published by Lippincott Williams \& Wilkins. 2011;118(2):201-8.

13. Lagatta J, Yan K, Hoffmann R. The association between 5-min Apgar score and mortality disappears after $24 \mathrm{~h}$ at the borderline of viability. Acta Paediatr. 2012;101(6):e243-7.

14. Kornacka MK, Musialik-Swietlinska E, Swietlinski J, et al. Usefulness of the Apgar score: a national survey of Polish neonatal centers. Ginekol Pol. 2011;82:39-43 PubMed .

15. Ephraim Ghide, Makonnen Assefa, Seid Mohammed, et al. Analysis of Deliveries in Jimma Hospital: A Four YearRetrospective Study. The Ethiopian Journal of Health Development. 1991;5(1):3-7.

16. Cacciani L, Asole S, Polo A, et al. Perinatal outcomes among immigrant mothers over two periods in a region of central Italy. BMC Public Health 2011;11 (294).

17. Ondoa-Onama C., Tumwine J. Immediate outcome of babies with low Apgar score in Mulago Hospital, Uganda. East African Medical Journal. 2003; 80(1).

18. Kilsztajn S, de Souza Lopes E, Nunes do Carmo MS, de Andrade AM. Apgar score associated with mode of delivery in São Paulo State, Brazil. Cad Saude Publica. 2007; 23(8):1886-92.

19. Olusanya BO, OA S. Correlates of birth asphyxia using two Apgar score classification methods. Nig Q J Hosp Med. 2010; 20(4):153-61. PubMed

20. American College of Obstetricians and Gynecologists. ACOG Practice Bulletin No. 106: Intrapartum fetal heart rate monitoring: nomenclature, interpretation, and general management principles. Obstet Gynecol 2009; 114:192.

21. Porreco RP, Clark SL, Belfort MA, et al. The changing specter of uterine rupture. Am J Obstet Gynecol 2009; 200:269.e1.

22. Zayeri F, Kazemnejad A, Ganjali M, et al. Incidence and risk factors of neonatal hypothermia at referral hospitals in Tehran, Islamic Republic of Iran. East Mediterr Health J. 2007;13(6):1308-18. PubMed

23. de Oliveira TG, Freire PV, Moreira FT, et al. Apgar score and neonatal mortality in a hospital located in the southern area of São Paulo City, Brazil. Einstein (Sao Paulo). 2012 Jan-Mar;10(1):22-8.

24. Mandy GT. Small for gestational age infant: complications In: Weisman LE, editor. Up-to-date 19.3. 2011.

25. Central Statistical Agency: ICF International. Ethiopia Demographic and Health Survey. Addis Ababa, Ethiopia, Calverton, Maryland, USA; 2001.

26. Central Statistical Agency: ICF International. Ethiopia Demographic and Health Survey. Addis Ababa, Ethiopia, Calverton, Maryland, USA; 2006.

27. Uganda Bureau of Statistics (UBOS), ORC Macro. Uganda Demographic and Health Survey 2000-2001. Calverton, Maryland, USA: UBOS and ORC Macro, 2001. 